\title{
Research on the File Date Transportation Based on ZigBee Protocol Jiaqiong Gao ${ }^{1}$ and Zhongsheng Wang ${ }^{2}$ \\ ${ }^{1}$ Department of Computer Science, Sichuan Vocational and Technical College Sichuan Suining 629000 \\ ${ }^{2}$ School of Computer Science and Engineering, Xi'an Technology University Xi'an 710021, China 516719510@qq.com
}

Keywords: ZigBee; Data merging; Stop-and-wait protocol

\begin{abstract}
This paper implements file transfer between two computers based on the characters of ZigBee that it works at low cost, low rate and short distance. It introduces the design of each hardware node and software module, moreover, the ZigBee wireless network, related protocols (such as ARQ) and technique of package and unpack ensure correct data-transfer. Through experimental testing, the correct file transfer between two computers is possible in the case of a small amount of data.
\end{abstract}

\section{Introduction}

Currently, files can be transferred correctly in the range of wireless network except the base station occurs some problems which may cause network paralyzed. ZigBee network could avoid this problem because it uses a Gateway node to enable other nodes to join its network directly or in a multi-hop way.

ZigBee, which is a low cost, short-delay, safe, close and other characteristics of the wireless sensor network communication technology, has been widely applied to the automatic control, industrial control, smart home, inventory management and other areas. In order to meet the cost-minimization requirements, this paper presents a file-transfer system based on ZigBee. Although ZigBee owns some defect such as low bandwidth and transmission rate, the file whose data amount is not large could correctly transfer in this network.

\section{System Design}

The system consists of upper and lower machine, the PC handles man-machine interface and file, lower machine mainly deals with file transfer. The system's routing node and gateway node are made up of ZigBee CC2530 Kit which not only supports 2.4ghz IEEE802.15.4 / ZigBee standard practice, but also can set up a strong-performance network.

The PC Works. The upper machine PC processes the data to be transmitted receives data and displays the user interface. All of these are based on visual c ++ 6.0 and MS Com technique. The MS Com control transmits and receives data through the serial port to provide a serial communication capacity for the application, which is a simplified Windows serial-communication programming Active control supported by Microsoft. This Active provides two methods for dealing with communication problems: event driven method (this paper used) and query method. Event driven is a very effective method to deal with the serial port interaction. In many cases, the event is required to be notified when some event occurs. For example, MS Com control's On Com event is able to capture and handle these communication events that a character arrives or an event occurs in a serial port and Carrier Detect (CD) or Request to Send (RTS) line receive a character or an event. Then we can handle these events to realize some function in the On Com event handler. The advantage of this method is that the program responds in time, the reliability is high, and each MS Com control corresponds to a serial port.

The Lower Machine. In this paper, DTK Company's CC2530 suite is used as the lower machine contains three kinds of nodes: terminal node, router node, central node. The ZigBee module can be 
setup into the required type. In the case, the terminated which is mobile node and sender PC are connected with RS232 serial line and the PC transmits the files to mobile terminated node through S232 serial line after processes these files ; the router which is a fixed node receives data from the terminal node, whose location should be suitable for the file transmission ; the central node is connected to the receiving end computer through the RS232 serial port, which is used to receive the data transmitted from the routing node and transmit data to PC to complete data decoding, merge, and other operation. The operator can get related information through UI and determine next step.

Software Implementation. In this paper, the system of file transmission is composed of three modules: data processing module, ZigBee data transmission module, data selection and saving module. As shown in Fig. 1.

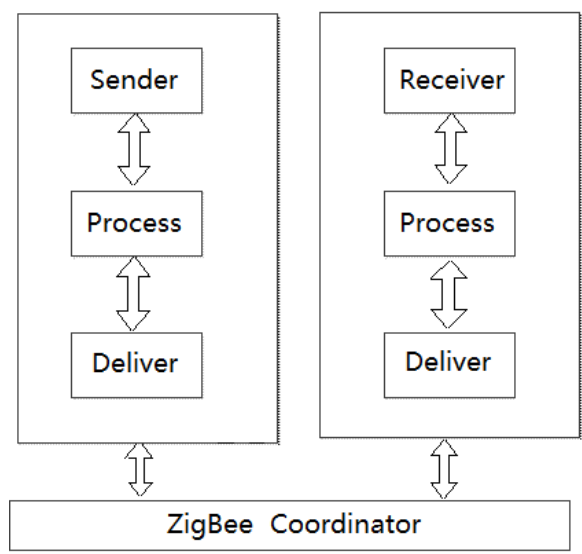

Figure 1. The diagram of file transfer process module

Program Design of Data Processing Module. The data processing module programmed by the Visual C++ is used to process the transmitted data and ensure the integrity and correctness of the ZigBee wireless network. In the general, the compressed file contains hundreds of bytes even tens of thousands of bytes, which may cause the loss of bytes in case of direct transmission through ZigBee, then sub-package of large file is essential. According to theory and experience, the size of a packet is set to 73 bytes, the file which is less than 73 bytes will be directly transmitted and others will be carried out the packet transmission (the packet is name as the pattern of "source file name" + "-" +"serial number". It is obvious that the shorter the time of file transmission, the smaller the packet, the longer the transmission time. So, the size of package should refer to practical demand for the case of higher timeliness requirements, file should be divided into some large packets and for the higher accuracy requirements, packet can be divided into small packets.

Program Design of ZigBee Data Transmission Module. ZigBee data transmission module is designed to realize the function of data transmission. Firstly, the file is divided into many renamed packets if necessary and these packets could transmit to ZigBee module which is connected to PC by RS232 serial port. Then, the packets disseminate in the ZigBee network. These packet data would be transmitted to the routing node through the terminal node, and finally arrive to the central node. The central node receives the packet data in order and delivers data to PC through the RS232 serial line. Finally, the PC would handle these data such as decoding, merge, storage and so on.

Program Design of Data Selection and Save Module. Data selection and preservation is realized by humanized user interface programmed with $\mathrm{C}++$, moreover, the man-machine interface lets people who are not familiar with the system operate system in short time.

When sending some data, the first step is to import the certain file required to be transmitted in UI, the file name could display in UI edit-box if correctly selected. Then choose decent compression algorithm to condense the file and divide it into renamed packets, by the way, these packets should be send into ZigBee network in order according the packet name. On the other hand, the receiver computer receives the uploaded sub-packets in sequence and compares serial number of the received packet with that of the local receiving state variable. If the two is equal, receiver PC 
should receive the new data packet and send confirmation message, otherwise received message should be discarded. After the process of decompression, preservation, consolidation and reference to packets' name, the data packet could be restored to a integrated file.

In this process, the file-receiving progress will display on receiver PC and we can view and edit the received integrated files if necessary.

\section{Data Frame Format}

ZigBee network is a network which is created by the central coordinator. When a node joins, the node would be assigned network address .In this way, we can form a variety of network (such as star network, tree network and network) topology by ZigBee network according to practical demands.

ZigBee network supports the transmission of data between any nodes in the network, and the data transmission between nodes has characters of transparent and point-to-point. The main point of this paper is point- to- point transmission. In this pattern, receiver and sender should have good agreement which would ensure a correct data transmission between points. Point-to-point data transmission has three frame formats. The first pattern "ZigBee short address" whose instruction format is" 0xFD+ data length + destination address + source-data", the recipient receives all the data and the address of the source. The second pattern is to use ZigBee short address to locate receiver by setting the sender, and the recipient will remove the packets' header and tail .The third pattern is similar to the second except for the addressing mode: the latter use custom address instead of "ZigBee short address".

\section{Key Technologies}

This paper adopts the key technologies of sub-package and combination of the file in two PCs, which has the advantages of low cost, simple operation, etc.

Sub-Package Process. The first step is to compress the selected file, then calculate the size of compressed file. The file whose size is no more than preset (in this paper, the preset size is 73 byte) could be transmitted directly, otherwise file should be divided into data packets according to preset size. The detail of sub-package is that :firstly, the file name(whose size is also no more than the upper bound of the packet 73 bytes) is placed in the first packet for transmission to notify the receiver which file will be send then the source file should be divide into some 73-byte packets and renamed by the pattern of < "source file name" + "-" + "the serial number $i>$.According to above mode, we can complete sub-package of files smoothly and save the packets in the certain folder which is ready to be transmitted sequentially into ZigBee network through serial line.

Stop Wait Protocol. Packets of files should be transmitted sequentially. In order to guarantee the correct of the data transmission, the recipient computer sends a confirmation message after receiving the packet. In the other side, sender confirms whether the packet is received successfully and determines the necessity of re-transmit packet. If necessary, the packet should re-transmit several times in the range of upper bound . The next packet could be send after the former is correctly received. Above process is known as the stop waiting protocol.

Stop waiting protocol is that a packet of file transmits through the ZigBee wireless sensor network to the receiver. In this process, only receiving the correct serial number of confirmation message, the sender could send a next new packet, or the packet would be re-transmitted in certain range until it is correctly received. If the sender receives a confirmation message with the same serial number, it is indicated that the receiving end receives the error packet which may be repeated, wrong order or loss packet. At this time, the data packets should be sent again, until the sender receives the correct confirmation message. The notability is that the frequency of re-transmission is limited to 5.If after retrying five times the sender still does not receive the correct confirmation message of transmitted packet, the whole file rather than the single packet should be re-send and the PC will display tips "Failed to send" in UI.

Merging Files. The receiving end will save the packets as small files whose name is in 
accordance with the packet name. The files which has the same prefix of name would be stored in the folder and be merged into a large file according to the serial number. After completion of large file, the related small files would be removed.

\section{System Testing}

There are two ZigBee communication modules in this system, and each of which connects with computer through RS232 serial line. The necessity of setting up routing node, which can function as a Repeater, is determined by distance of two computers. If computers are close, terminal node and central node can complete the test of system. After the system is powered up, the function of data transmission between the two computers through the star-pattern ZigBee network.

The UI of this system provides the function of selecting files and displaying related information of process. This system mainly realizes two functions. First, it is regarded as a chat tool which could provide capability of IM. Besides, it can transmit file data between two computers. The main topic of this paper is the later function file-transmission. File transfer interface as shown in Fig. 2.

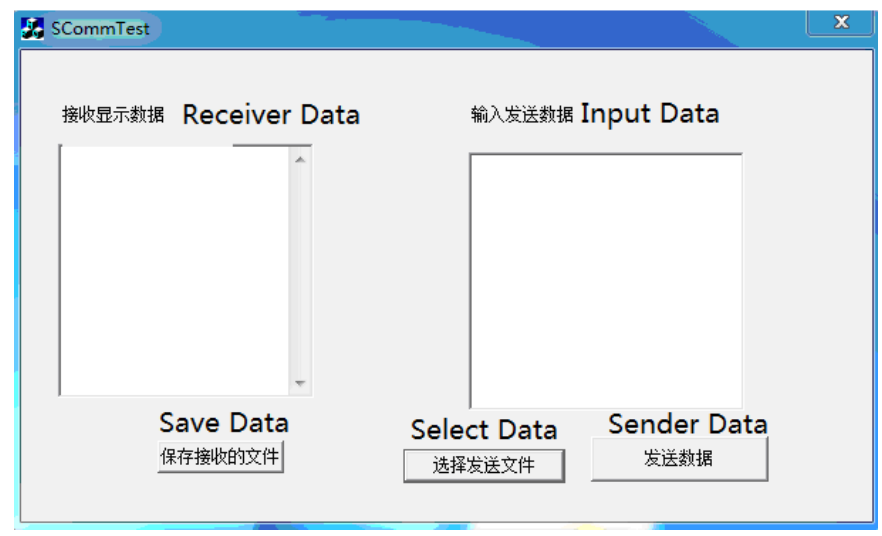

Figure 2. File transfer interface

The specific practices of testing are as follows: Taking the notebook and desktop as two computer terminals. When clicking the "select send file" button in sending end computer, the "open" dialog box appears in which we can select file or input some data in edit box. The abstract of file or data displays in sender edit box. When clicking the "send data" button, the data is transmitted to receiving end computer through ZigBee wireless network .After some delay; we find some data are received in receiving end computer displayed in the suitable edit box. we can click "save" button to preserve data as file, and some tips display if the operation is success, as shown in Fig. 3 and Fig. 4.So far, the whole works of testing is completed.

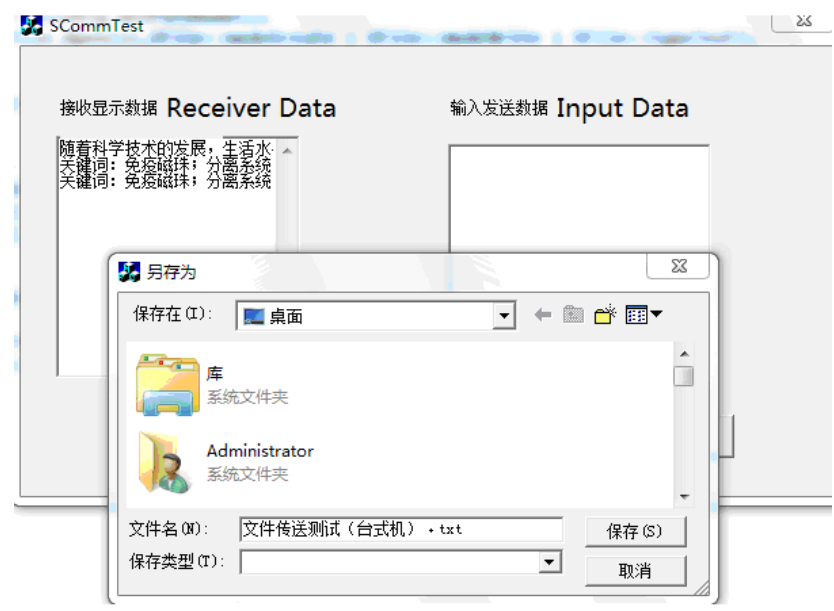

Figure 3. Saving the received data 


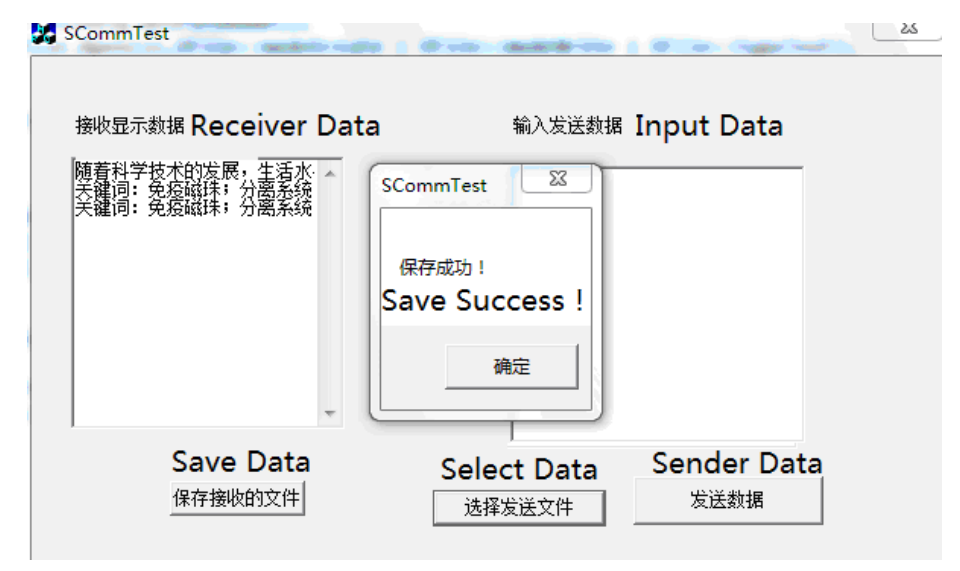

Figure 4. Saving success

\section{Conclusion}

This paper presents a file transfer system based on ZigBee between two computers. Through the system design, this paper expounds the working process of the whole system which presents hardware and software, data frame format, key technology and system testing. Hardware design includes the choice of hardware and the realization, Software design includes data processing and UI design between ZigBee network and computer; the format of data frame is the point-to- point data of ZigBee network; the key technology is sub-package, stop waiting protocol for and file merge. This system is based on the simple and flexible ZigBee wireless network, which has the characteristics of low cost, simple hardware and software programming.

\section{References}

[1] Z.Wang, D.L.Huang, Research on Key Technology of Image Transmission Based on ZigBee, Computer Technology and Development, 2015.05, pp183-186.

[2] W. Z.S, Research on the Network Layer Topology Measurement Based on SNMP, AJETR 2013-02, pp123-126

[3] Xiao Shang Research of AS level Network Topology, AJETR-2015-02,pp 109-123

[4] Pei, Donghui, The Design and Implementation of Serial Monitoring Software Based on ZigBee, ICICEE2012.08, pp236-241

[5] Holman R,Stanley J, O zkan-Haller T. Applying video sensor networks to nearshore environment monitoring[J].IEEE Trans. on Pervasive Computing, 2003, 2(4); 14-21.

[6] Akyildiz I F, Melodia T, Chowdhury K R. A survey on wireless multimedia sensor networks [J]. Computer Networks, 2007, 51 ( 4 ):

[7] Z.S., D.L. H, AMR, Research And Implementation Of Image Transmission Key Technological Based On WSN, PP 4759-4762, 2014.

[8] Chen Guoshao, The Design of Greenhouse Environment Monitoring System Based on ZigBee, AJETR2014-01, PP86 -91. 